Supporting Information

\title{
A facile and green combined strategy for improving photocatalytic activity of carbon nitride
}

Xueping Song, Dan Tang, Yefeng Chen, Mengyun Yin, Qin Yang, Zeqin Chen, Limei Zhou*

Chemical Synthesis and Pollution Control Key Laboratory of Sichuan Province, China West Normal University, Nanchong 637002, Sichuan, China. E-mail: cwnuzhoulimei@163.com 


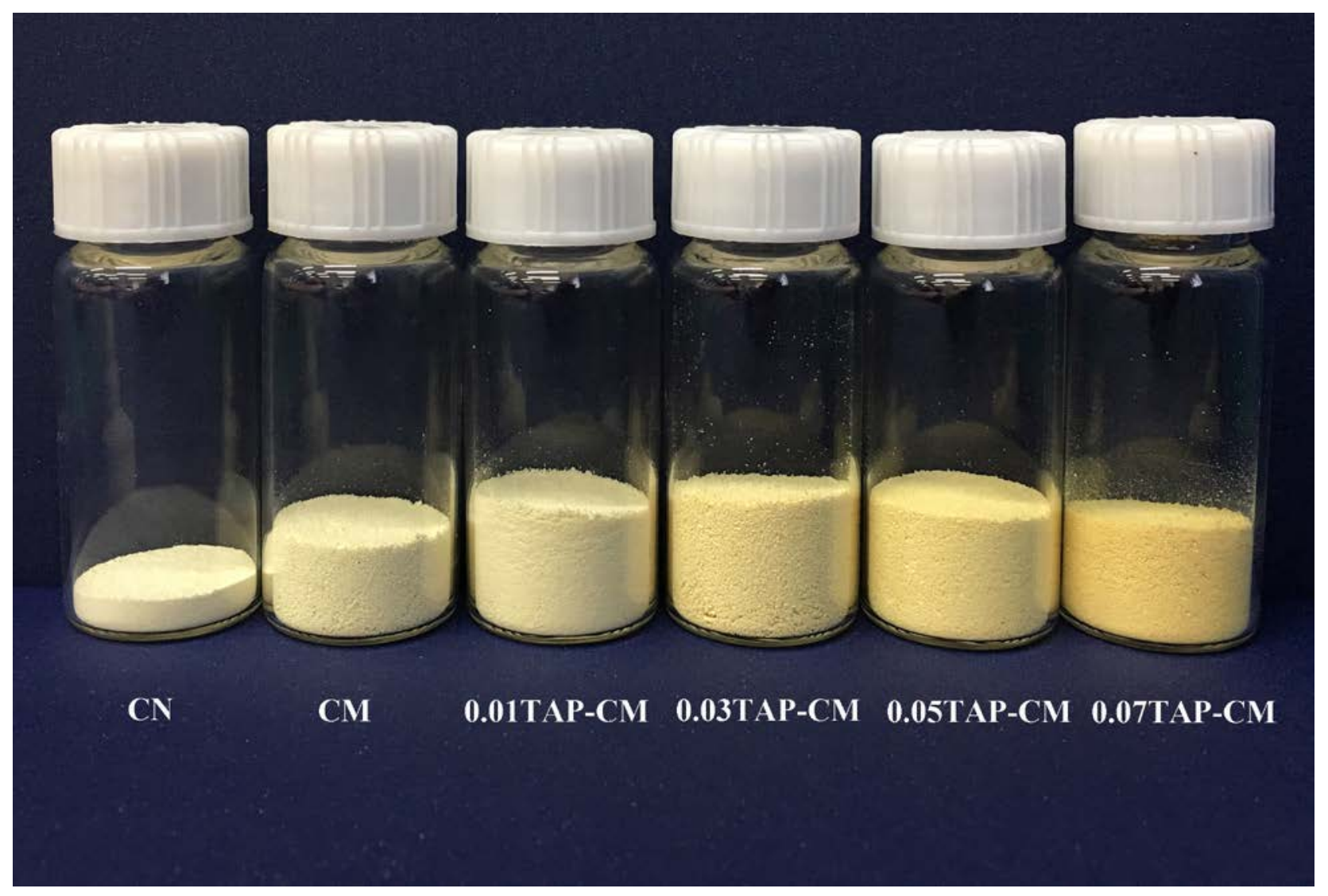

Figure S1. The photograph of different samples (0.2 g). 


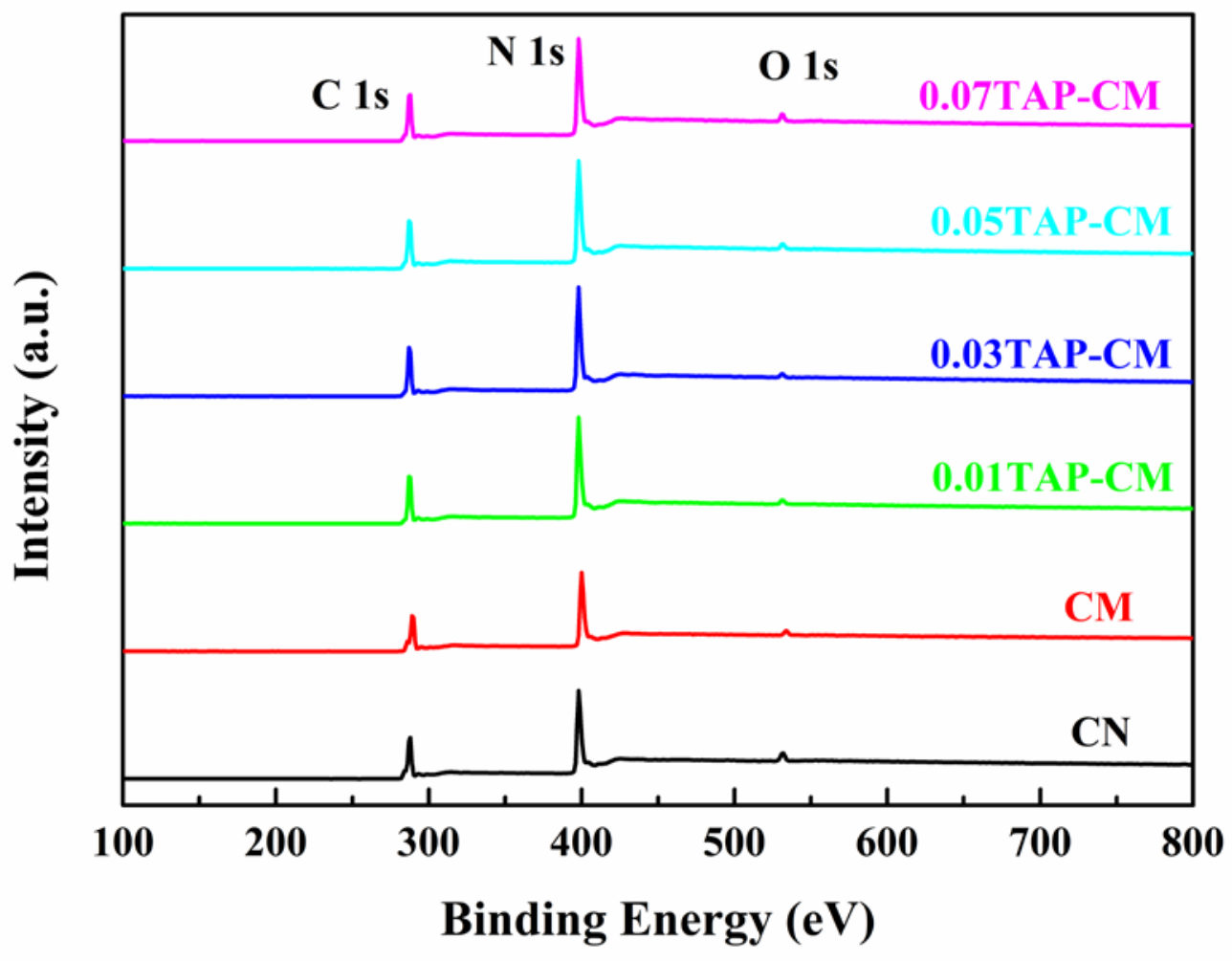

Figure S2. XPS survey spectrum of different samples. 

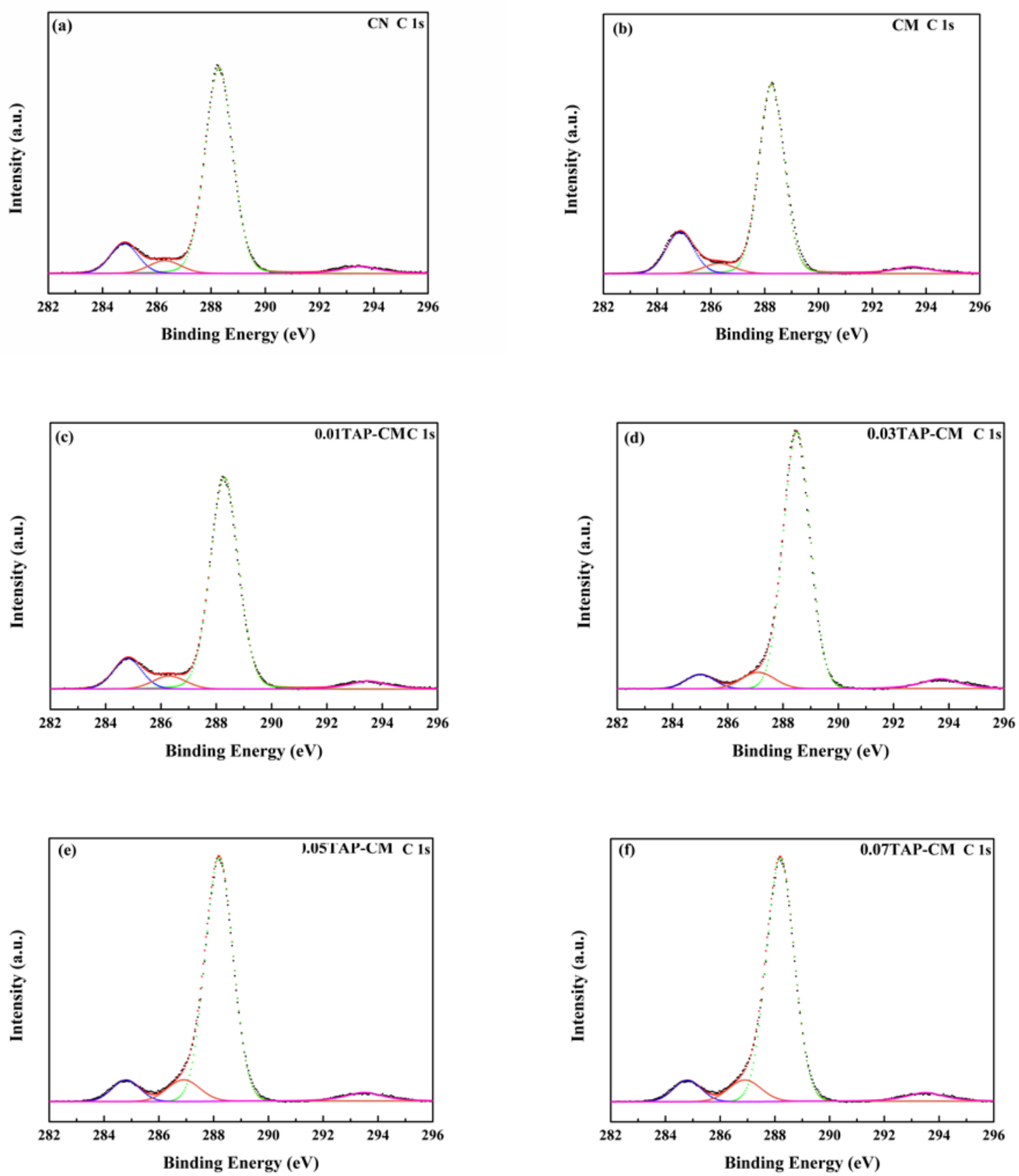

Figure S3. High resolution C 1s XPS spectrum of CN (a), CM (b), 0.01TAP-CM (c), 0.03TAP-CM (d), 0.05TAP-CM (e), and 0.07TAP-CM (f). 


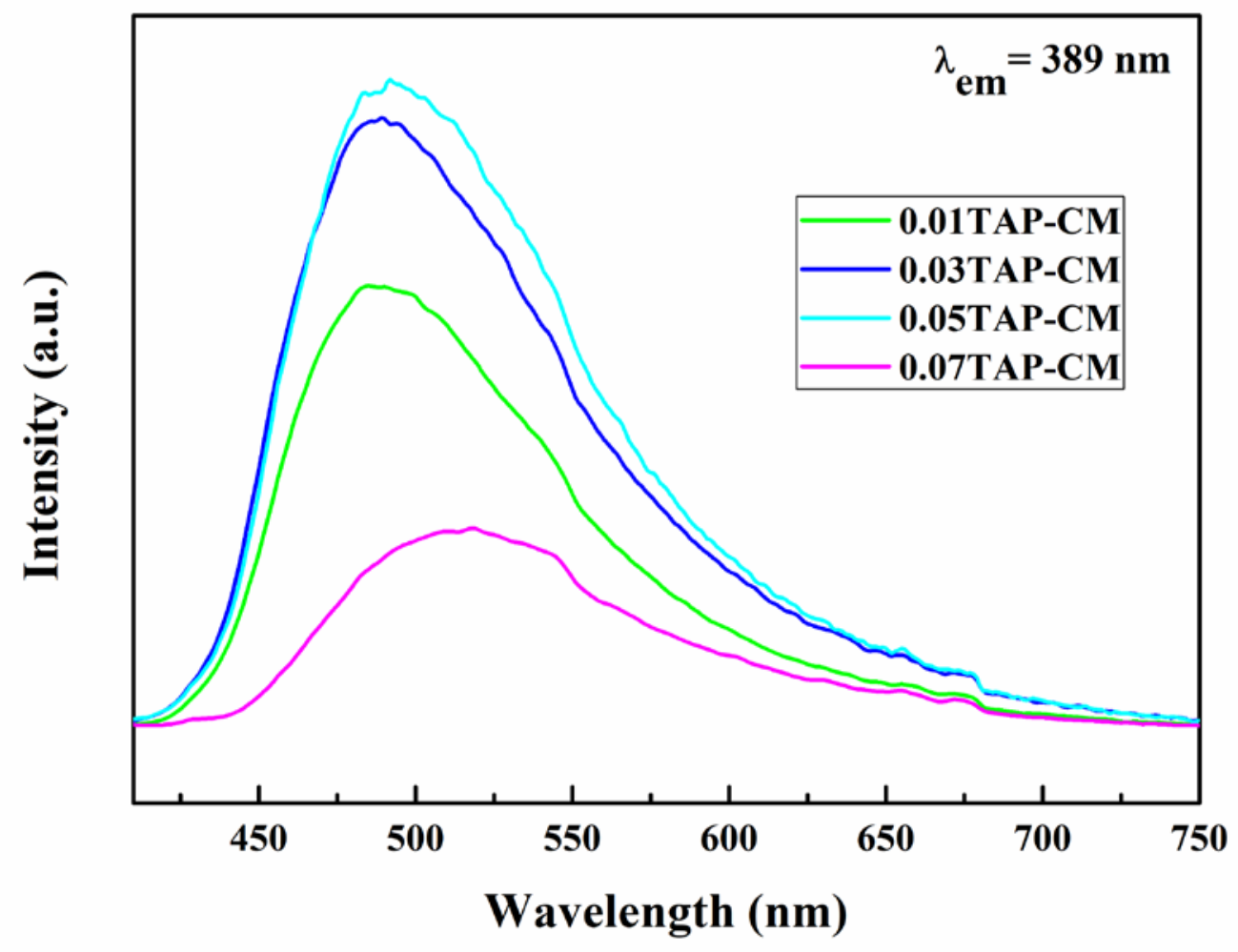

Figure S4. PL spectra of 0.01TAP-CM, 0.03TAP-CM, 0.05TAP-CM, and 0.07TAP-CM. 